Discussion Paper \# 2009-09

Continuity of the value and optimal strategies when common priors change

by

Ezra Einy, Ori Haimanko and Biligbaatar Tumendemberel

July 2009 


\title{
Continuity of the value and optimal strategies when common priors change
}

\author{
Ezra Einy, Ori Haimankoł Biligbaatar Tumendemberel ${ }^{\ddagger}$
}

July 2009

\begin{abstract}
We show that the value of a zero-sum Bayesian game is a Lipschitz continuous function of the players' common prior belief, with respect to the total variation metric (that induces the topology of setwise convergence on beliefs). This is unlike the case of general Bayesian games, where lower semi-continuity of Bayesian equilibrium payoffs rests on the convergence of conditional beliefs (Engl (1994), Kajii and Morris (1998)). We also show upper, and approximate lower, semicontinuity of the optimal strategy correspondence with respect to the total variation norm, and discuss approximate lower semi-continuity of the Bayesian equilibrium correspondence in the context of zero-sum games.

JEL Classification Number: C72.

Keywords: Zero-Sum Bayesian Games, Common Prior, Value, Optimal Strategies, Upper Semi-Continuity, Lower Approximate SemiContinuity.
\end{abstract}

*Graduate School of Economics, Hitotsubashi University, Naka 2-1, Kunitachi, Tokyo 186-8601, Japan; and Department of Economics, Ben-Gurion University of the Negev, Beer Sheva 84105, Israel. E-mail: einy@bgu.ac.il

${ }^{\dagger}$ JSPS Fellow, Graduate School of Economics, Hitotsubashi University, Naka 2-1, Kunitachi, Tokyo 186-8601, Japan; and Department of Economics, Ben-Gurion University of the Negev, Beer Sheva 84105, Israel. E-mail: orih@bgu.ac.il

${ }^{\ddagger}$ Graduate School of Economics, Hitotsubashi University, Naka 2-1, Kunitachi, Tokyo 186-8601, Japan. E-mail: em071007@g.hit-u.ac.jp 


\section{Introduction}

Bayesian games describe situations where there is uncertainty about players' payoffs, and players may have different private information about the realized state of nature that affects the payoffs. Being a natural framework for modelling numerous real world issues, it has been a subject of extensive investigation in the literature. In particular, the question of continuity of Bayesian equilibria (BE) with respect to changes in players' information endowments received some attention.

One strand of research concentrated on the continuity of BE with respect to changes in information partitions, or fields (see, e.g., Monderer and Samet (1996), Einy et al (2008)). Another strand considered the effects of small changes in the players' common prior belief (see, e.g., Milgrom and Weber (1985), Engl (1995), and Kajii and Morris (1994, 1998)). Milgrom and Weber (1985) showed upper semi-continuity (USC) of the BE correspondence under a very general condition, that requires that the common prior be sufficiently "spread-out" on the product of players' types. This condition is satisfied trivially in the important case where each player has at most countably many types, which is equivalent to assuming that his private information is given by a countable partition of the space of states of nature. In this latter framework, Engl (1995) investigated (approximate) lower semicontinuity $((A) L S C)$ of the BE expected payoff correspondence, under the uniform setwise convergence topology on priors.

The ALSC means that for any $\mathrm{BE}$ in a game and any $\varepsilon>0$, there is an $\varepsilon$-equilibrium with close expected payoffs in the same game, for any close enough common prior. ${ }^{1}$ Engl (1995) showed that the BE expected payoff correspondence is ALSC, assuming that the approximating $\varepsilon$-BE are with respect to ex-ante expected utilities. However, if players evaluate the consequences of their strategic choices at the interim stage, following the receipt of private information, they are in fact concerned with their interim expected utility, that takes into account their private information and is based on the correspondingly updated prior belief. But while ex-ante and interim $\mathrm{BE}$ are the same, this is not true for the approximate, $\varepsilon$-BE, since an exante $\varepsilon$-best response may be hugely suboptimal for some realizations of the player's private information, albeit with small probability. Kajii and Morris

\footnotetext{
${ }^{1}$ The stronger notion of lower semi-continuity $(L S C)$ requires that the $\mathrm{BE}$ expected payoff be approximable by expected payoffs of true BE in games with close enough common priors.
} 
$(1994,1998)$ showed that, if the approximate $\varepsilon$-BE are taken in the interim sense, ALSC of the BE expected payoff and strategy correspondences may fail if priors are converging only setwise. They showed that to obtain ALSC of the interim BE expected payoff correspondence, uniform across bounded games, it is necessary (and sufficient) to additionally assume almost uniform convergence of beliefs conditional on players' private information (i.e., that the closeness of conditional beliefs becomes approximate common knowledge with high ex-ante probability).

In this work we consider zero-sum Bayesian games. These games recently came into spotlight, particularly in the context of comparing information structures and measuring the value of information (see, e.g., Gossner and Mertens (2001), Lehrer and Rosenberg (2006)). We start by showing that the value of a zero-sum game is a Lipschitz continuous function of players' common prior belief, with respect to the total variation metric on the set of priors; see Theorem 1. (This metric induces the setwise convergence topology on priors.)

Although being in line with Engl's (1994) result on the ALSC of the ex-ante BE expected payoff correspondence, Theorem 1 implies a previously unnoticed fact. Since pairs of optimal strategies are both interim and ex-ante $\mathrm{BE}$ in a zero-sum Bayesian game, and the value (三the expected BE payoff) is a continuous function of the common prior, the interim $\mathrm{BE}$ expected payoff correspondence is in fact LSC (and in particular ALSC) when restricted to zero-sum games. Thus, the assumptions of Kajii and Morris (1998) on the convergence of conditional beliefs, which are necessary for ALSC in the nonzero-sum setting, are not needed in the context of zero-sum Bayesian games.

Theorem 1 bears semblance to another uniform continuity result for the value of zero-sum games, in Einy et al (2008), which was established in a different setting: the common prior of the players was fixed, but their information fields were variable, and the set of fields was endowed with the Boylan pseudo-metric. This result does not imply Theorem 1, however, as the latter deals with variable common priors.

We further show that the optimal strategy correspondence is both USC (Proposition 1) and ALSC (Proposition 2) with respect to the total variation metric on priors. Since optimal strategies are both ex-ante and interim BE strategies in zero-sum Bayesian games, Proposition 1 implies that the ex-ante and the interim BE correspondences are USC. However, the notion of ALSC uses $\varepsilon$-optimal strategies to approximate the given optimal strategy, and $\varepsilon$ optimal strategies are defined with respect to the ex-ante expected payoffs in 
the game. Thus Proposition 2 implies that the ex-ante BE correspondence is ALSC in zero-sum Bayesian games, but it sheds no light on the ALSC of the interim $\mathrm{BE}$ correspondence in these games.

As was mentioned, the interim BE correspondence may not be ALSC with respect to the total variation metric on priors, in cases where conditional beliefs do not converge almost uniformly (see Kajii and Morris (1994)). Our last two results show that in some circumstances the interim BE correspondence is ALSC in zero-sum Bayesian games without any assumptions on the convergence of conditional beliefs. Proposition 3 identifies one such instance in games where each player has an infinite information partition; the main assumption is that knowledge of player's own type allows him to guess the type of the other player while making a bounded error. When at least one of the players has a finite information partition, ALSC of the interim BE correspondence obtains without this asssumption, see Proposition 4.

The paper is organized as follows. The setup is described in section 2 and our results are stated and proved in section 3.

\section{Preliminaries}

\subsection{Zero-Sum Bayesian Games}

We consider zero-sum games with two players, $i=1,2$. Games are played in an uncertain environment, which affects payoff functions of the players. The underlying uncertainty is described by a probability space $(\Omega, \mathcal{F}, \mu)$, where $\Omega$ is a set of states of nature, $\mathcal{F}$ is a $\sigma$-field of events in $\Omega$, and $\mu$ is a countably additive probability measure on $(\Omega, \mathcal{F})$ that represents the common prior belief of the players about the distribution of the realized state of nature. The information endowment of player $i$ is given by an (at most) countable and $\mathcal{F}$-measurable partition $\Pi^{i}$ of $\Omega$. Given $\omega \in \Omega$, denote by $\Pi^{i}(\omega)$ the element of the partition $\Pi^{i}$ that contains $\omega$. If $\omega$ was realized, player $i$ only knows that the realized state of nature belongs to $\Pi^{i}(\omega)$.

Each player $i=1,2$ has a set $S^{i}$ of strategies, which is a convex and 
compact subset of a Euclidean space ${ }^{2}$. Additionally, there is a measurable ${ }^{3}$ real valued payoff function $u: \Omega \times S^{1} \times S^{2} \rightarrow \mathbb{R}$. At every state of nature $\omega \in \Omega, u\left(\omega, s^{1}, s^{2}\right)$ is the payoff received by player 1 , and $-u\left(\omega, s^{1}, s^{2}\right)$ is the payoff of player 2, when each player $i$ chooses to play $s^{i}$. We assume that, at every $\omega \in \Omega$, each player's payoff is continuous and concave in his own strategy; that is, $u\left(\omega, \cdot, s^{2}\right)$ is continuous and concave for a fixed $s^{2} \in S^{2}$, and $u\left(\omega, s^{1}, \cdot\right)$ is continuous and convex for a fixed $s^{1} \in S^{1}$. We further assume that $|u|$ is bounded on $\Omega \times S^{1} \times S^{2}$ by some $M>0$ (Remark 1 below explains the necessity of this assumption).

The probability space $(\Omega, \mathcal{F}, \mu)$, information endowments $\Pi^{1}$ and $\Pi^{2}$, strategy sets $S^{1}$ and $S^{2}$, and the payoff function $u$ fully describe a zero-sum Bayesian game. To concentrate on the effects of changes in the common prior, we keep all the attributes of the game fixed henceforth, with the exception of $\mu \in \Delta(\Omega, \mathcal{F}) \equiv$ the set of all countably additive probability measures on $(\Omega, \mathcal{F})$. For any $\mu \in \Delta(\Omega, \mathcal{F})$, the associated zero-sum Bayesian game will be denoted by $G(\mu)$.

A Bayesian strategy of player $i$ is a $\Pi^{i}$-measurable function $x^{i}: \Omega \rightarrow S^{i}$, i.e., $x^{i}$ is constant on any $\pi^{i} \in \Pi^{i}$. The set of all Bayesian strategies of player $i$ will be denoted by $X^{i}$. Clearly, $X^{i}$ can be identified with the function space $\left(S^{i}\right)^{\Pi^{i}}$, which is convex and compact in the product topology, and also metrizable in it since $\Pi^{i}$ is at most countable. Given $\mu \in \Delta(\Omega, \mathcal{F})$, the expected payoff of player 1 (and the expected loss of player 2) when $x^{i} \in X^{i}$ is chosen by $i=1,2$ is

$$
U_{\mu}\left(x^{1}, x^{2}\right) \equiv \int_{\Omega} u\left(\omega, x^{1}(w), x^{2}(w)\right) d \mu(\omega) .
$$

Remark 1. In order for the expected payoff function $U_{\mu}$ to be well defined for a given $\mu \in \Delta(\Omega, \mathcal{F}), \mu$-integrability of an $\mathcal{F}$-measurable

$$
f(\omega) \equiv \sup _{\left(s^{1}, s^{2}\right) \in S^{1} \times S^{2}}\left|u\left(\omega, s^{1}, s^{2}\right)\right|
$$

\footnotetext{
${ }^{2}$ All our results, with the exception of Proposition 3, use only the fact that $S^{i}$ is a compact and metrizable subset of a topological vector space. For Proposition 3, the assumption of $S^{i}$ being a Banach space, not necessarily of finite dimension, would have sufficed. We, however, confine ourselves to the finite-dimension framework, so as to avoid unnecessary generality.

${ }^{3}$ The measurability is with respect to the $\sigma$-field $\mathcal{F}$ in the first coordinate, and with respect to the Borel $\sigma$-fields in the second and third coordinates.
} 
would have sufficed, without the need to assume uniform boundedness of $u$ as we did earlier. However, since our interest lies in changing common priors in the game with a fixed utility function, $f$ needs to be integrable with respect to all $\mu \in \Delta(\Omega, \mathcal{F})$. This, in fact, implies the existence of $M=\sup _{\omega \in \Omega} f(\omega)<\infty$.

With our assumptions on $u$, the expected payoff function $U_{\mu}$ is continuous and concave in $x^{1} \in X^{1}$ for a fixed $x^{2} \in X^{2}$, and continuous and convex in $x^{2} \in X^{2}$ for a fixed $x^{1} \in X^{1}$ (the continuity is implied by the bounded convergence theorem). Thus, Sion minimax theorem (see, e.g., Theorem A.7 in Sorin (2002)) guarantees existence of the value $v(\mu)$ in each game $G(\mu)$ : the following inequality holds,

$$
\min _{x^{2} \in X^{2}} \max _{x^{1} \in X^{1}} U_{\mu}\left(x^{1}, x^{2}\right)=\max _{x^{1} \in X^{1}} \min _{x^{2} \in X^{2}} U_{\mu}\left(x^{1}, x^{2}\right),
$$

and $v(\mu)$ is defined as the common value of the two expressions in (1).

Given $\varepsilon \geq 0, \widehat{x}^{1} \in X^{1}$ is called $\varepsilon$-optimal for player 1 in $G(\mu)$ if

$$
U_{\mu}\left(\widehat{x}^{1}, x^{2}\right) \geq v(\mu)-\varepsilon
$$

for any $x^{2} \in X^{2}$. Similarly, $\widehat{x}^{2} \in X^{2}$ is called $\varepsilon$-optimal for player 2 in $G(\mu)$ if

$$
U_{\mu}\left(x^{1}, \widehat{x}^{2}\right) \leq v(\mu)+\varepsilon
$$

for any $x^{1} \in X^{1}$. If a strategy $x^{i}$ is 0 -optimal for player $i$, it is called optimal for $i$. The set of $\varepsilon$-optimal strategies of player $i$ in $G(\mu)$ will be denoted by $\mathcal{O}_{\varepsilon}^{i}(\mu)$. It is convex and compact. The notation for $\mathcal{O}_{0}^{i}(\mu)$, the set of optimal strategies, will be simplified to $\mathcal{O}^{i}(\mu)$.

Optimality of a strategy is closely related to the concept of equilibrium. A pair $\left(\widehat{x}^{1}, \widehat{x}^{2}\right) \in X^{1} \times X^{2}$ is called an ex-ante Bayesian $\varepsilon$-equilibrium (henceforth $\varepsilon$-EBE for short) if

$$
U_{\mu}\left(\widehat{x}^{1}, \widehat{x}^{2}\right) \geq U_{\mu}\left(x^{1}, \widehat{x}^{2}\right)-\varepsilon
$$

for any $x^{1} \in X^{1}$, and

$$
U_{\mu}\left(\widehat{x}^{1}, \widehat{x}^{2}\right) \leq U_{\mu}\left(\widehat{x}^{1}, x^{2}\right)+\varepsilon
$$

for any $x^{2} \in X^{2}$. Denote by $E B E_{\varepsilon}(\mu)$ the set of all $\varepsilon$-EBE in $G(\mu)$, and simplify $E B E_{0}(\mu)$ to $E B E(\mu)$. If $\left(\widehat{x}^{1}, \widehat{x}^{2}\right) \in E B E(\mu)$, we will call it an ex-ante Bayesian equilibrium ( $E B E$ for short). 
Remark 2. Note that, for every $\varepsilon \geq 0$,

$$
\mathcal{O}_{\varepsilon}^{1}(\mu) \times \mathcal{O}_{\varepsilon}^{2}(\mu) \subset E B E_{2 \varepsilon}(\mu)
$$

and

$$
E B E_{\varepsilon}(\mu) \subset \mathcal{O}_{2 \varepsilon}^{1}(\mu) \times \mathcal{O}_{2 \varepsilon}^{2}(\mu) .
$$

In particular,

$$
\mathcal{O}^{1}(\mu) \times \mathcal{O}^{2}(\mu)=E B E(\mu),
$$

and the value $v(\mu)$ is the unique EBE payoff (to player 1) in the game $G(\mu)$.

Example 1 (Matrix Bayesian Game). Assume that each player $i$ has $n_{i}$ pure strategies, and $S^{i}$ is the $\left(n_{i}-1\right)$-dimensional simplex of $i$ 's mixed strategies. Assume further that in each $\omega \in \Omega$, the payoff function is given by

$$
u\left(\omega, s^{1}, s^{2}\right)=s^{1} A(\omega) s^{2},
$$

where strategy $s^{1} \in S^{1}$ is regarded as a row vector, $s^{2} \in S^{2}$ - as a column vector, and $A(\omega)$ is an $n_{1} \times n_{2}$-matrix, with $A(\omega)_{j, k}$ being the payoff of player 1 when he chooses pure strategy $j$ and 2 - pure strategy $k$, which is uniformly bounded across $\Omega$. Then the strategy sets of players and the payoff function satisfy all the conditions listed above, and the associated zero-sum Bayesian game is amenable to our analysis.

\subsection{Interim Expected Payoffs}

The notions of the value of a game, and of the optimality of strategies, are defined with respect to players' ex-ante expected payoffs. In other words, players are assumed to evaluate their utilities before any private information is revealed. However, they may conceivably want to evaluate the consequences of their strategic choices at the interim stage, following the receipt of private information. In other words, players may be concerned with their interim expected payoff, that takes into account their private information and is based on the appropriately updated prior belief.

To formalize the discussion, let $\mu \in \Delta(\Omega, \mathcal{F})$. For any $\omega \in \Omega$ and $i=1,2$, denote by $\Pi^{i}(\omega)$ the element of partition $\Pi^{i}$ that contains $\omega$. If $\mu\left(\Pi^{i}(\omega)\right)>$ 
0 , denote by $\mu_{\Pi^{i}(\omega)} \in \Delta(\Omega, \mathcal{F})$ the conditional belief of player $i$, given his information at $\omega$, i.e., for any $A \in \mathcal{F}$,

$$
\mu_{\Pi^{i}(\omega)}(A)=\mu\left(A \mid \Pi^{i}(\omega)\right)=\frac{\mu\left(A \cap \Pi^{i}(\omega)\right)}{\mu\left(\Pi^{i}(\omega)\right)} .
$$

The function $U_{\mu_{\Pi^{i}(\omega)}}(\cdot, \cdot)$ will be referred to as the interim expected payoff given $\Pi^{i}(\omega)$.

For $\varepsilon \geq 0$, a pair $\left(\widehat{x}^{1}, \widehat{x}^{2}\right) \in X^{1} \times X^{2}$ is called an interim Bayesian $\varepsilon$ equilibrium (henceforth, $\varepsilon-I B E$ for short) in $G(\mu)$ if

$$
U_{\mu_{\Pi^{1}(\omega)}}\left(\widehat{x}^{1}, \widehat{x}^{2}\right) \geq U_{\mu_{\Pi^{1}(\omega)}}\left(x^{1}, \widehat{x}^{2}\right)-\varepsilon
$$

for every $x^{1} \in X^{1}$ and every $\omega \in \Omega$ with $\mu\left(\Pi^{1}(\omega)\right)>0$, and

$$
U_{\mu_{\Pi^{2}(\omega)}}\left(\widehat{x}^{1}, \widehat{x}^{2}\right) \leq U_{\mu_{\Pi^{2}(\omega)}}\left(\widehat{x}^{1}, x^{2}\right)+\varepsilon
$$

for every $x^{2} \in X^{2}$ and every $\omega \in \Omega$ with $\mu\left(\Pi^{2}(\omega)\right)>0$. Denote by $I B E_{\varepsilon}(\mu)$ the set of all $\varepsilon$-IBE in $G(\mu)$, and simplify $I B E_{0}(\mu)$ to $I B E(\mu)$. If $\left(\widehat{x}^{1}, \widehat{x}^{2}\right) \in$ $I B E(\mu)$, we will call it an interim Bayesian equilibrium (IBE for short).

Remark 3. When $\varepsilon=0$, there is no distinction between IBE and EBE. Definitions embodied in (2), (3) and (6), (7) are equivalent, as are indeed the notions of IBE and EBE in general, non-zero-sum, games. Accordingly, neither the value of a zero-sum Bayesian game (viewed as the ex-ante payoff in an IBE) nor the optimal strategies (viewed as IBE strategies) need not be redefined in the interim expected payoffs setting.

Remark 4. When $\varepsilon>0$, the definition of $\varepsilon$-IBE is significantly more demanding than that of $\varepsilon$-EBE. Although any $\varepsilon$-IBE is in particular an $\varepsilon$ EBE, i.e., $I B E_{\varepsilon}(\mu) \subset E B E_{\varepsilon}(\mu)$, as follows from integrating both sides in (6) and (7) over $\Omega$, the opposite is not true. In terms of the interim expected payoffs $U_{\mu_{\Pi^{i}(\omega)}}(\cdot, \cdot)$, the definition of $\left(\widehat{x}^{1}, \widehat{x}^{2}\right) \in E B E_{\varepsilon}(\mu)$ implies that

$$
U_{\mu_{\Pi^{1}(\omega)}}\left(\widehat{x}^{1}, \widehat{x}^{2}\right) \geq U_{\mu_{\Pi^{1}(\omega)}}\left(x^{1}, \widehat{x}^{2}\right)-\frac{\varepsilon}{\mu\left(\Pi^{1}(\omega)\right)}
$$

for every $x^{1} \in X^{1}$ and every $\omega \in \Omega$ with $\mu\left(\Pi^{1}(\omega)\right)>0$, and

$$
U_{\mu_{\Pi^{2}(\omega)}}\left(\widehat{x}^{1}, \widehat{x}^{2}\right) \leq U_{\mu_{\Pi^{2}(\omega)}}\left(\widehat{x}^{1}, x^{2}\right)+\frac{\varepsilon}{\mu\left(\Pi^{2}(\omega)\right)}
$$


for every $x^{2} \in X^{2}$ and every $\omega \in \Omega$ with $\mu\left(\Pi^{2}(\omega)\right)>0$. This indicates that although an $\varepsilon$-EBE strategy $\widehat{x}^{i}$ is ex-ante an $\varepsilon$-best response against $\widehat{x}^{j}$, it may be hugely interim-suboptimal in states of nature $\omega$ with low probability $\mu\left(\Pi^{i}(\omega)\right)$, thereby failing to be an $\varepsilon^{\prime}$-IBE strategy for all sufficiently small $\varepsilon^{\prime}$. (See, e.g., Example 2 in section 3.3.)

\subsection{Topology on Common Priors}

Consider the total variation metric $d$ on $\Delta(\Omega, \mathcal{F})$, given by

$$
d\left(\mu, \mu^{\prime}\right)=\sup _{E \in \mathcal{F}}\left|\mu(E)-\mu^{\prime}(E)\right|
$$

for any $\mu, \mu^{\prime} \in \Delta(\Omega, \mathcal{F})$.

The following lemma shows that the expected payoff $U_{\mu}$ is a Lipschitz function of $\mu$ with respect to $d$, for a fixed $\left(x^{1}, x^{2}\right) \in X^{1} \times X^{2}$.

Lemma 1. For any $\left(x^{1}, x^{2}\right) \in X^{1} \times X^{2}$ and $\mu, \mu^{\prime} \in \Delta(\Omega, \mathcal{F})$,

$$
\left|U_{\mu}\left(x^{1}, x^{2}\right)-U_{\mu^{\prime}}\left(x^{1}, x^{2}\right)\right| \leq 2 M d\left(\mu, \mu^{\prime}\right)
$$

Proof. For any $\mu, \mu^{\prime} \in \Delta(\Omega, \mathcal{F})$,

$$
\sup \int_{\Omega} f(\omega) d\left(\mu-\mu^{\prime}\right)(\omega)=2 d\left(\mu, \mu^{\prime}\right),
$$

where the supremum is taken over all $\mathcal{F}$-measurable functions $f: \Omega \rightarrow[-1,1]$ (see, e.g., Lemma 1 on p. 360 in Shiryaev (1996)). Given any $\left(x^{1}, x^{2}\right) \in$ $X^{1} \times X^{2}$, note that, by the boundedness of $u$ and (11),

$$
\begin{aligned}
\left|U_{\mu}\left(x^{1}, x^{2}\right)-U_{\mu^{\prime}}\left(x^{1}, x^{2}\right)\right|= & \mid \int_{\Omega} u\left(\omega, x^{1}(\omega), x^{2}(\omega)\right) d \mu(\omega) \\
& -\int_{\Omega} u\left(\omega, x^{1}(\omega), x^{2}(\omega)\right) d \mu^{\prime}(\omega) \mid \\
= & \left|\int_{\Omega} u\left(\omega, x^{1}(\omega), x^{2}(\omega)\right) d\left(\mu-\mu^{\prime}\right)(\omega)\right| \\
\leq & 2 M d\left(\mu, \mu^{\prime}\right) .
\end{aligned}
$$




\section{Results}

\subsection{Continuity of Value}

Our main result establishes Lipschitz continuity of the value:

Theorem 1. The value $v(\mu)$ is a Lipschitz continuous function of $\mu$ with respect to the metric $d$ : for any $\mu, \mu^{\prime} \in \Delta(\Omega, \mathcal{F})$,

$$
\left|v(\mu)-v\left(\mu^{\prime}\right)\right| \leq 2 M d\left(\mu, \mu^{\prime}\right)
$$

Proof. Let $\hat{x}^{1} \in \mathcal{O}^{1}(\mu)$ and $x^{2} \in X^{2}$. The optimality of $\hat{x}^{1}$ in $G(\mu)$ and Lemma 1 imply that

$$
\begin{aligned}
U_{\mu^{\prime}}\left(\hat{x}^{1}, x^{2}\right) & \geq U_{\mu}\left(\hat{x}^{1}, x^{2}\right)-2 M d\left(\mu, \mu^{\prime}\right) \\
& \geq v(\mu)-2 M d\left(\mu, \mu^{\prime}\right) .
\end{aligned}
$$

This holds for every $x^{2} \in X^{2}$, and hence it follows that

$$
\begin{aligned}
v\left(\mu^{\prime}\right) & =\max _{x^{1} \in X^{1}} \min _{x^{2} \in X^{2}} U_{\mu^{\prime}}\left(x^{1}, x^{2}\right) \\
& \geq \min _{x^{2} \in X^{2}} U_{\mu^{\prime}}\left(\hat{x}^{1}, x^{2}\right) \\
& \geq v(\mu)-2 M d\left(\mu, \mu^{\prime}\right) .
\end{aligned}
$$

Similarly, starting with $\hat{x}^{2} \in \mathcal{O}^{2}(\mu)$ we obtain

$$
\begin{aligned}
v\left(\mu^{\prime}\right) & =\min _{x^{2} \in X^{2}} \max _{x^{1} \in X^{1}} U_{\mu^{\prime}}\left(x^{1}, x^{2}\right) \\
& \leq \max _{x^{1} \in X^{1}} U_{\mu^{\prime}}\left(x^{1}, \hat{x}^{2}\right) \\
& \leq v(\mu)+2 M d\left(\mu, \mu^{\prime}\right)
\end{aligned}
$$

The combination of (13) and (14) yields (12).

Theorem 1 implies, in particular, that the single-valued EBE (or, equivalently via Remark 3, IBE) expected payoff correspondence is both lower and upper semi-continuous with respect to the total variation metric on the 
common prior, when restricted to zero-sum Bayesian games. This stands in contrast to the general, non-zero-sum case. As was shown by Kajii and Morris (1998), IBE payoffs in a non-zero-sum Bayesian game $G(\mu)$ may be quite far from $\varepsilon$-IBE payoffs in $G\left(\mu^{\prime}\right)$ for all small enough $\varepsilon>0$, even if $d\left(\mu, \mu^{\prime}\right)$ is arbitrarily small, when the beliefs conditional on each player's private information do not converge uniformly. But, in the zero-sum case, the IBE payoff in $G(\mu)$ is approximated by the true IBE payoff in $G\left(\mu^{\prime}\right)$ (not merely an $\varepsilon$-IBE payoff) when $d\left(\mu, \mu^{\prime}\right) \rightarrow 0$.

\subsection{Upper Semi-continuity of Optimal Strategies}

In addition to the value of a zero-sum Bayesian game, optimal strategies also have strong continuity properties with respect to the total variation metric on the common prior. Given a sequence $\left\{\mu_{n}\right\}_{n=0}^{\infty} \subset \Delta(\Omega, \mathcal{F})$ such that $\lim _{n \rightarrow \infty} \mu_{n}=\mu_{0}$, we say that the optimal strategy correspondence is upper semi-continuous (USC) along $\left\{\mu_{n}\right\}_{n=1}^{\infty}$ if the following holds: for any player $i$ and any sequence $\left\{\hat{x}_{n}^{i}\right\}_{n=1}^{\infty} \subset X^{i}$ such that $\lim _{n \rightarrow \infty} \hat{x}_{n}^{i}=\hat{x}_{0}^{i}$ and $\hat{x}_{n}^{i} \in \mathcal{O}^{i}\left(\mu_{n}\right)$ for each $n \geq 1, \hat{x}_{0}^{i} \in \mathcal{O}^{i}\left(\mu_{0}\right)$.

Proposition 1. The optimal strategy correspondence is USC along any convergent sequence $\left\{\mu_{n}\right\}_{n=1}^{\infty} \subset \Delta(\Omega, \mathcal{F})$.

Proof. Let $\left\{\mu_{n}\right\}_{n=1}^{\infty} \subset \Delta(\Omega, \mathcal{F})$ and $\left\{\hat{x}_{n}^{i}\right\}_{n=1}^{\infty} \subset X^{i}$ be such that $\lim _{n \rightarrow \infty} \mu_{n}=$ $\mu_{0}, \lim _{n \rightarrow \infty} \hat{x}_{n}^{i}=\hat{x}_{0}^{i}$, and $\hat{x}_{n}^{i} \in O^{i}\left(\mu_{n}\right)$ for each $n \geq 1$. We will assume $i=1$, the case of $i=2$ being analogous. Take any $x^{2} \in X^{2}$. By assumption, for any $n \geq 1$

$$
U_{\mu_{n}}\left(\hat{x}_{n}^{1}, x^{2}\right) \geq v\left(\mu_{n}\right) .
$$

Using Lemma 1, we obtain

$$
\begin{aligned}
& \left|U_{\mu_{n}}\left(\hat{x}_{n}^{1}, x^{2}\right)-U_{\mu_{0}}\left(\hat{x}_{0}^{1}, x^{2}\right)\right| \\
\leq & \left|U_{\mu_{n}}\left(\hat{x}_{n}^{1}, x^{2}\right)-U_{\mu_{0}}\left(\hat{x}_{n}^{1}, x^{2}\right)\right|+\left|U_{\mu_{0}}\left(\hat{x}_{n}^{1}, x^{2}\right)-U_{\mu_{0}}\left(\hat{x}_{0}^{1}, x^{2}\right)\right| \\
\leq & 2 M d\left(\mu_{n}, \mu_{0}\right)+\left|U_{\mu_{0}}\left(\hat{x}_{n}^{1}, x^{2}\right)-U_{\mu_{0}}\left(\hat{x}_{0}^{1}, x^{2}\right)\right|,
\end{aligned}
$$

and thus

$$
\lim _{n \rightarrow \infty} U_{\mu_{n}}\left(\hat{x}_{n}^{1}, x^{2}\right)=\lim _{n \rightarrow \infty} U_{\mu_{0}}\left(\hat{x}_{n}^{1}, x^{2}\right)=U_{\mu_{0}}\left(\hat{x}_{0}^{1}, x^{2}\right)
$$


by continuity of $U_{\mu_{0}}$ in the first variable. Now, taking the limits of both sides in (15) and using Theorem 1 yields

$$
U_{\mu_{0}}\left(\hat{x}_{0}^{1}, x^{2}\right) \geq v\left(\mu_{0}\right) .
$$

Since this holds for every $x^{2} \in X^{2}, \hat{x}_{0}^{1} \in \mathcal{O}^{i}\left(\mu_{0}\right)$.

Since $\mathcal{O}^{1}(\mu) \times \mathcal{O}^{2}(\mu)=E B E(\mu)$ for every $\mu \in \Delta(\Omega, \mathcal{F})$, as was mentioned in Remark 2, Proposition 1 also establishes that the EBE correspondence (and, equivalently by Remark 3, the IBE correspondence) is USC in zero-sum Bayesian games.

\subsection{Approximate Lower Semi-continuity of Optimal Strate- gies, EBE, and IBE}

Defining lower semi-continuity of the optimal strategy correspondence requires some care. Its straightforward version will not work: given $\left\{\mu_{n}\right\}_{n=0}^{\infty} \subset$ $\Delta(\Omega, \mathcal{F})$ with $\lim _{n \rightarrow \infty} \mu_{n}=\mu_{0}$ and $\hat{x}_{0}^{i} \in \mathcal{O}^{i}\left(\mu_{0}\right)$, we may not be able to find a sequence $\left\{\hat{x}_{n}^{i}\right\}_{n=1}^{\infty} \subset X^{i}$ such that $\lim _{n \rightarrow \infty} \hat{x}_{n}^{i}=\hat{x}_{0}^{i}$ and $\hat{x}_{n}^{i}$ is optimal in $G\left(\mu_{n}\right)$ for each $n \geq 1$. Indeed, even in a simple decision problem (i.e., a oneplayer game) not all payoff maximizers may be approximable by maximizers in nearby problems. Thus, the appropriate notion of lower semi-continuity of the optimal strategy correspondence with respect to the common prior is the following. We will say that the optimal strategy correspondence is approximately lower semi-continuous $\left(A L S C\right.$ ) along a sequence $\left\{\mu_{n}\right\}_{n=1}^{\infty}$ with $\lim _{n \rightarrow \infty} \mu_{n}=\mu_{0}$ if the following holds: Given any $\hat{x}_{0}^{i} \in \mathcal{O}^{i}\left(\mu_{0}\right)$ for some player $i$, and any $\varepsilon>0$, there exists a sequence $\left\{\hat{x}_{n}^{i}\right\}_{n=1}^{\infty} \subset X^{i}$ with $\lim _{n \rightarrow \infty} \hat{x}_{n}^{i}=\hat{x}_{0}^{i}$, such that $\hat{x}_{n}^{i}$ is (merely) $\varepsilon$-optimal in $G\left(\mu_{n}\right)$ for every $n \geq 1$.

Similarly, the EBE (respectively, IBE) correspondence is defined to be $A L S C$ along a sequence $\left\{\mu_{n}\right\}_{n=1}^{\infty}$ with $\lim _{n \rightarrow \infty} \mu_{n}=\mu_{0}$ by the requirement that, given any $\left(\hat{x}^{1}, \hat{x}^{2}\right) \in E B E\left(\mu_{0}\right)$ (respectively, $\left.I B E\left(\mu_{0}\right)\right)$ and any $\varepsilon>0$, there exists a sequence $\left\{\left(\hat{x}_{n}^{1}, \hat{x}_{n}^{2}\right)\right\}_{n=1}^{\infty} \subset X^{1} \times X^{2}$ with $\lim _{n \rightarrow \infty}\left(\hat{x}_{n}^{1}, \hat{x}_{n}^{2}\right)=$ $\left(\hat{x}_{0}^{1}, \hat{x}_{0}^{2}\right)$, such that $\left(\hat{x}_{n}^{1}, \hat{x}_{n}^{2}\right) \in E B E_{\varepsilon}\left(\mu_{n}\right)$ (respectively, $\left.I B E_{\varepsilon}\left(\mu_{n}\right)\right)$ for every $n \geq 1$.

It follows from the next proposition that the optimal strategy correspondence is ALSC, with the sequence $\left\{\hat{x}_{n}^{i}\right\}_{n=1}^{\infty}$ being the strategy $\hat{x}_{0}^{i}$ itself: 
Proposition 2. For every $\mu, \mu^{\prime} \in \Delta(\Omega, \mathcal{F})$ and every $i=1,2$,

$$
\mathcal{O}^{i}(\mu) \subset \mathcal{O}_{4 M d\left(\mu, \mu^{\prime}\right)}^{i}\left(\mu^{\prime}\right) .
$$

Proof. As in the proof of Proposition 1, we will only consider the case of $i=1$. Fix any $\mu \in \Delta(\Omega, \mathcal{F})$ and let $\hat{x}^{1} \in \mathcal{O}^{1}(\mu)$. By Lemma 1 , optimality of $\hat{x}^{1}$, and Theorem 1 , for any $x^{2} \in X^{2}$ and any $\mu^{\prime} \in \Delta(\Omega, \mathcal{F})$,

$$
\begin{aligned}
U_{\mu^{\prime}}\left(\hat{x}^{1}, x^{2}\right) & \geq U_{\mu}\left(\hat{x}^{1}, x^{2}\right)-2 M d\left(\mu, \mu^{\prime}\right) \\
& \geq v(\mu)-2 M d\left(\mu, \mu^{\prime}\right) \\
& \geq v\left(\mu^{\prime}\right)-4 M d\left(\mu, \mu^{\prime}\right) .
\end{aligned}
$$

This shows that $\hat{x}^{1}$ is indeed $4 M d\left(\mu, \mu^{\prime}\right)$-optimal for player 1 in $G\left(\mu^{\prime}\right)$.

According to Proposition 2, if $\lim _{n \rightarrow \infty} \mu_{n}=\mu_{0}$ then, for a given $\varepsilon>0$, any $\hat{x}_{0}^{i}$ which is optimal in $G\left(\mu_{0}\right)$ is also $\varepsilon$-optimal in $G\left(\mu_{n}\right)$ for all sufficiently large $n$. However, while optimality of a strategy has an interpretation in terms of both ex-ante and interim expected payoffs (since $\mathcal{O}^{1}(\mu) \times \mathcal{O}^{2}(\mu)=E B E(\mu)$ $=I B E(\mu)$, by Remarks 2 and 3$)$, this is no longer so with $\varepsilon$-optimality which is a purely ex-ante concept (as expounded in Remark 4). Thus, although Proposition 2 trivially implies that the EBE correspondence is ALSC along any converging sequence $\left\{\mu_{n}\right\}_{n=1}^{\infty}$ (since $E B E\left(\mu_{0}\right)=\mathcal{O}^{1}\left(\mu_{0}\right) \times \mathcal{O}^{2}\left(\mu_{0}\right)$ and $E B E_{\varepsilon}\left(\mu_{n}\right) \supset \mathcal{O}_{\frac{\varepsilon}{2}}^{1}\left(\mu_{n}\right) \times \mathcal{O}_{\frac{\varepsilon}{2}}^{2}\left(\mu_{n}\right)$ by Remark 2), it remains mute on IBE. And indeed, a pair $\left(\hat{x}^{1}, \hat{x}^{2}\right) \in \mathcal{O}^{1}\left(\mu_{0}\right) \times \mathcal{O}^{2}\left(\mu_{0}\right)=I B E\left(\mu_{0}\right)$ may fail to be in $I B E_{\varepsilon}\left(\mu_{n}\right)$ for all $n \geq 1$ and all small enough $\varepsilon$ :

Example 2. Let $\Omega=\mathbb{N}$ (the set of positive integers), $S^{1}=[0,1]$, $S^{2}=\{0\}, \Pi^{1}=\Pi^{2} \equiv\{\{2 n, 2 n-1\}: n \in \mathbb{N}\}$, and, finally,

$$
u\left(\omega, s^{1}, s^{2}\right) \equiv\left\{\begin{array}{cc}
-\left(s^{1}\right)^{2}, & \text { if } \omega \text { is odd } \\
0, & \text { is } \omega \text { is even. }
\end{array} .\right.
$$

If $\mu_{0}$ is a probability measure with the full support on $\{2 n: n \in \mathbb{N}\}$, then, clearly, $\hat{x}_{0}^{1}(\cdot) \equiv 1$ is an optimal strategy of player 1 in $G\left(\mu_{0}\right)$. Consider, however, a sequence $\left\{\mu_{n}\right\}_{n=1}^{\infty}$ of probability measure, such that, for every 
$n, \mu_{n}$ is identical to $\mu_{0}$ on all subsets of $\Omega \backslash\{2 n, 2 n-1\}$, but $\mu_{n}(\{2 n\})=$ $\mu_{n}(\{2 n-1\})=\frac{1}{2} \mu_{0}(\{2 n\})$. Then clearly $\lim _{n \rightarrow \infty} \mu_{n}=\mu_{0}$, but $\left(\hat{x}_{0}^{1}, 0\right) \notin$ $I B E_{\varepsilon}\left(\mu_{n}\right)$ for every $n \geq 1$ and all $\varepsilon \in\left[0, \frac{1}{2}\right)$.

However, the failure of some $\left(\hat{x}_{0}^{1}, \hat{x}_{0}^{2}\right) \in I B E\left(\mu_{0}\right)$ to be in $I B E_{\varepsilon}\left(\mu_{n}\right)$ for all small enough $\varepsilon>0$, does not rule out that the IBE correspondence is ALSC: it does not preclude the possibility that $\left(\hat{x}_{0}^{1}, \hat{x}_{0}^{2}\right)$ is approximable by a sequence $\left\{\left(\hat{x}_{n}^{1}, \hat{x}_{n}^{2}\right)\right\}_{n=1}^{\infty}$ with $\left(\hat{x}_{n}^{1}, \hat{x}_{n}^{2}\right) \in I B E_{\varepsilon}\left(\mu_{n}\right)$ for each $n \geq 1$. Indeed, the $\operatorname{IBE}\left(\hat{x}_{0}^{1}, 0\right)$ in Example 2 is the limit of the sequence $\left\{\left(\hat{x}_{n}^{1}, 0\right)\right\}_{n=1}^{\infty}$, where $\hat{x}_{n}^{1}(\{2 k, 2 k-1\}) \equiv\left\{\begin{array}{cc}1, & \text { if } k \neq n, \\ 0, & \text { if } k=n\end{array}\right.$ for all $k \in \mathbb{N}$, and $\left\{\left(\hat{x}_{n}^{1}, 0\right)\right\}_{n=1}^{\infty}$ are $\varepsilon$ IBE (and even IBE) in $\left\{G\left(\mu_{n}\right)\right\}_{n=1}^{\infty}$. It is therefore natural to ask under what conditions the IBE correspondence is ALSC in general zero-sum Bayesian games.

It is well known that the IBE correspondence in non-zero-sum Bayesian games is not ALSC with respect to the total variation metric on $\Delta(\Omega, \mathcal{F})$. It is shown in Kajii and Morris (1994) (Example 1, Section 4.2 there) that an IBE in a non-zero-sum Bayesian game $G(\mu)$ may be far from all $\varepsilon$-IBE in $G\left(\mu_{n}\right)$, for all sufficiently low values of $\varepsilon$, and in all states of nature. What is at fault in that example is a non-uniform (across $\Omega$ ) convergence of prior beliefs conditional on players' private information (i.e., of measures $\left.\left(\left(\mu_{n}\right)_{\Pi^{i}(\omega)}\right)_{i=1,2 ; \omega \in \Omega}\right)$, which may occur despite that $\lim _{n \rightarrow \infty} \mu_{n}=\mu_{0}$ in the total variation metric (see also Example 2 above). More precisely, the problem lies in the lack of almost uniform convergence of conditional beliefs, which is defined, roughly speaking, by the requirement that the closeness of conditional beliefs becomes approximate common knowledge with high ex-ante probability. ${ }^{4}$ Our next Proposition 3 indicates that the (almost) uniform convergence of conditional beliefs may not be necessary for the IBE correspondence being ALSC in zero-sum Bayesian games. The proposition makes certain assumptions on the support of $\left\{\mu_{n}\right\}_{n=0}^{\infty}$ that guarantee ALSC behavior along this sequence with respect to the total variation metric, without

\footnotetext{
${ }^{4}$ The notion of almost uniform convergence of conditional beliefs is defined and expounded upon in Kajii and Morris (1994). We do not attempt to give a formal definition here, since this would require a lengthy digression, and this notion's relevance is primarily for the issue of IBE expected payoff continuity in general (non-zero-sum) games, which is not our focus.
} 
any additional requirement on the convergence of conditional beliefs. ${ }^{5}$

Before we state Proposition 3, the following convention is in order. For each $i=1,2$, we will write the elements of the (at most countable) partition $\Pi^{i}$ as an indexed sequence $\left\{\pi_{j}^{i}\right\}_{j=1}^{T^{i}}$, where $T^{i} \in\{\infty\} \cup \mathbb{N}$. When $i$ 's private information is given by $\pi_{j}^{i}$ (i.e., when the realized state of nature $\omega$ is such that $\Pi^{i}(\omega)=\pi_{j}^{i}$ ), the index $j$ of $\pi_{j}^{i}$ may be referred to as the type of player $i$.

Proposition 3. Let $\left\{\mu_{n}\right\}_{n=0}^{\infty} \subset \Delta(\Omega, \mathcal{F})$ be such that $\lim _{n \rightarrow \infty} \mu_{n}=\mu_{0}$, and assume that in zero-sum Bayesian games $\left\{G\left(\mu_{n}\right)\right\}_{n=0}^{\infty}$ :

(I) there exists $C>0$ such that, for every $\omega \in \Omega$, the payoff function $u(\omega, \cdot, \cdot)$ is Lipschitz continuous with a constant $C$ with respect to the Euclidean norm on $S^{1} \times S^{2}$;

(II) $T^{1}=T^{2}=\infty$, and there exist functions $t^{1}, t^{2}: \mathbb{N} \rightarrow \mathbb{N}$ with $\lim _{n \rightarrow \infty} \min \left(t^{1}, t^{2}\right)(n)=\infty$, and an integer $K>0$ such that, for every $i=1,2$ and every $j \geq 1$ :

(a) $\left(\mu_{0}\right)\left(\pi_{j}^{i}\right)>0$

and

(b) for every $n \geq 0$, if $\left(\mu_{n}\right)\left(\pi_{j}^{i}\right)>0$ then the measure $\left(\mu_{n}\right)_{\pi_{j}^{i}}$ has a $t^{i}(j)+K$

support on the set $\bigcup_{k=t^{i}(j)} \pi_{k}^{-i}$, where $-i$ denotes the rival of player $i$.

Then the IBE correspondence is ALSC along $\left\{\mu_{n}\right\}_{n=1}^{\infty}$.

Note that assumption (I) is implied by the uniform boundedness of $u$ if $\left\{G\left(\mu_{n}\right)\right\}_{n=0}^{\infty}$ are Bayesian matrix games (see Example 1). Assumption

\footnotetext{
${ }^{5}$ Indeed, under the assumptions of Proposition 3, the conditional beliefs of the players may not converge uniformly. Moreover, it is easy to find examples (in the spirit of Example 1 in Kajii and Morris (1994)) of $\left\{\mu_{n}\right\}_{n=0}^{\infty}$, adhereing to the assumptions, such that the weaker, almost uniform, convergence of conditional beliefs also fails.
} 
(IIb) means that although player $i$ may be unsure of the type of his rival, he can estimate it via the function $t^{i}$ and in doing so make an error of at most $K$ (given the knowledge of his own type). This assumption is satisfied, for instance, by the information structure in the electronic mail game of Rubinstein (1994).

Proof of Proposition 3. Let $\left\{\mu_{n}\right\}_{n=0}^{\infty} \subset \Delta(\Omega, \mathcal{F})$ be a sequence satisfying all the assumptions. Fix $\left(\hat{x}_{0}^{1}, \hat{x}_{0}^{2}\right) \in I B E\left(\mu_{0}\right)$ and $\varepsilon>0$. Consider, additionally, a sequence $\left\{\left(\widehat{y}_{n}^{1}, \widehat{y}_{n}^{2}\right)\right\}_{n=1}^{\infty} \subset X^{1} \times X^{2}$ such that $\left(\widehat{y}_{n}^{1}, \widehat{y}_{n}^{2}\right) \in$ $\mathcal{O}^{1}\left(\mu_{n}\right) \times \mathcal{O}^{2}\left(\mu_{n}\right)$ for each $n \geq 1$. Let

$$
0<\delta \leq \delta_{0} \equiv \frac{\varepsilon}{8 C K^{2}\left(\max _{s^{1} \in S^{1}}\left\|s^{1}\right\|+\max _{s^{2} \in S^{2}}\left\|s^{2}\right\|\right)},
$$

where $\|\cdot\|$ denotes the Euclidean norm on both $S^{1}$ and $S^{2}$. Define a sequence $\left\{\left(\bar{x}_{n, \delta}^{1}, \bar{x}_{n, \delta}^{2}\right)\right\}_{n=1}^{\infty} \subset X^{1} \times X^{2}$ as follows: for every $n \geq 1, i=1,2$, and $j \geq 1$, set

$$
\bar{x}_{n, \delta}^{i}\left(\pi_{j}^{i}\right) \equiv \max (1-\delta j, 0) \widehat{x}_{0}^{i}\left(\pi_{j}^{i}\right)+\min (\delta j, 1) \widehat{y}_{n}^{i}\left(\pi_{j}^{i}\right) .
$$

Let $J(\delta) \geq\left[\frac{1}{\delta}\right]+1$ be such for all $j \geq J(\delta)$ and $i=1,2, t^{i}(j) \geq\left[\frac{1}{\delta}\right]+1$. Since $\lim _{n \rightarrow \infty} \mu_{n}=\mu_{0}$, it follows from assumption (IIa) on $\mu_{0}$ that there exists $N>0$ such that

$$
\varepsilon^{\prime} \equiv \frac{\varepsilon}{2} \inf \left\{\mu_{n}\left(\pi_{j}^{i}\right) \mid i \in\{1,2\} ; 1 \leq j \leq J(\delta) ; N \leq n\right\}>0 .
$$

By Proposition 2, the assumption that $\lim _{n \rightarrow \infty} \mu_{n}=\mu_{0}$, and the fact that $\left(\hat{x}_{0}^{1}, \hat{x}_{0}^{2}\right) \in \mathcal{O}^{1}\left(\mu_{0}\right) \times \mathcal{O}^{2}\left(\mu_{0}\right)$ (implied by the choice of $\left(\hat{x}_{0}^{1}, \hat{x}_{0}^{2}\right)$ and Remark 3 ), there exists $N(\delta) \geq N$ such that for every $n \geq N(\delta),\left(\hat{x}_{0}^{1}, \hat{x}_{0}^{2}\right) \in \mathcal{O}_{\varepsilon^{\prime}}^{1}\left(\mu_{n}\right) \times$ $\mathcal{O}_{\varepsilon^{\prime}}^{2}\left(\mu_{n}\right)$. We claim that $\left(\bar{x}_{n, \delta}^{1}, \bar{x}_{n, \delta}^{2}\right) \in I B E_{\varepsilon}\left(\mu_{n}\right)$ for each $n \geq N(\delta)$. We will only show that

$$
U_{\left(\mu_{n}\right)_{\pi_{j}^{1}}}\left(\bar{x}_{n, \delta}^{1}, \bar{x}_{n, \delta}^{2}\right) \geq U_{\left(\mu_{n}\right)_{\pi_{j}^{1}}}\left(x^{1}, \bar{x}_{n, \delta}^{2}\right)-\varepsilon
$$

for every $x^{1} \in X^{1}$, every $j \geq 1$, and every $n \geq N(\delta)$. It could then be established similarly that

$$
U_{\left(\mu_{n}\right)_{\pi_{j}^{2}}}\left(\bar{x}_{n, \delta}^{1}, \bar{x}_{n, \delta}^{2}\right) \leq U_{\left(\mu_{n}\right)_{\pi_{j}^{2}}^{2}}\left(\bar{x}_{n, \delta}^{1}, x^{2}\right)+\varepsilon
$$

for every $x^{2} \in X^{2}$, every $j \geq 1$, and every $n \geq N(\delta)$, and thus (6) and (7) in the definition of $\varepsilon$-IBE will follow. 
Given $x^{1} \in X^{1}$ and $j \geq 1$, consider two cases.

Case 1: $j \geq J(\delta)$. Note that, by (17) and the definition of $J(\delta)$, $\bar{x}_{n, \delta}^{1}(\omega)=\widehat{y}_{n}^{1}(\omega)$ for every $\omega \in \pi_{j}^{1}$ and $\bar{x}_{n, \delta}^{2}(\omega)=\widehat{y}_{n}^{2}(\omega)$ for every $\omega \in$ $t^{1}(j)+K$

$\bigcup_{k=t^{1}(j)} \pi_{k}^{2}$. Thus, by assumption (IIb),

$$
\left.U_{\left(\mu_{n}\right)_{\pi_{j}^{1}}^{1}} \bar{x}_{n, \delta}^{1}, \bar{x}_{n, \delta}^{2}\right)=U_{\left(\mu_{n}\right)_{\pi_{j}^{1}}^{1}}\left(\widehat{y}_{n}^{1}, \widehat{y}_{n}^{2}\right)
$$

and

$$
U_{\left(\mu_{n}\right)_{\pi_{j}^{1}}}\left(x^{1}, \bar{x}_{n, \delta}^{2}\right)=U_{\left(\mu_{n}\right)_{\pi_{j}^{1}}}\left(x^{1}, \widehat{y}_{n}^{2}\right) .
$$

But $\left(\widehat{y}_{n}^{1}, \widehat{y}_{n}^{2}\right) \in \mathcal{O}^{1}\left(\mu_{n}\right) \times \mathcal{O}^{2}\left(\mu_{n}\right)=I B E\left(\mu_{n}\right)$ (see Remarks 2 and 3). This fact and (21), (22) imply (19).

Case 2: $1 \leq j<J(\delta)$. Denote

$$
\overline{\bar{x}}_{n, \delta}^{1} \equiv \max (1-\delta j, 0) \widehat{x}_{0}^{1}+\min (\delta j, 1) \widehat{y}_{n}^{1}
$$

and

$$
\overline{\bar{x}}_{n, \delta}^{2} \equiv \max \left(1-\delta t^{1}(j), 0\right) \widehat{x}_{0}^{2}+\min \left(\delta t^{1}(j), 1\right) \widehat{y}_{n}^{2}
$$

(note a subtle but important difference between $\bar{x}_{n, \delta}^{i}$ and $\bar{x}_{n, \delta}^{i}-$ in the former the coefficients in the convex combination are state-dependent). It follows from the definition of $\bar{x}_{n, \delta}^{i}$ and $\overline{\bar{x}}_{n, \delta}^{i}$ and assumptions (I) and (II) that

$$
\left|U_{\left(\mu_{n}\right)_{\pi_{j}^{1}}}\left(\bar{x}_{n, \delta}^{1}, \bar{x}_{n, \delta}^{2}\right)-U_{\left(\mu_{n}\right)_{\pi_{j}^{1}}}\left(\overline{\bar{x}}_{n, \delta}^{1}, \overline{\bar{x}}_{n, \delta}^{2}\right)\right|
$$

(by assumption (IIb))

$\leq \sum_{k=t^{1}(j)}^{k=t^{1}(j)+K} \int_{\pi_{j}^{1} \cap \pi_{k}^{2}}\left|u\left(\omega, \bar{x}_{n, \delta}^{1}(w), \bar{x}_{n, \delta}^{2}(w)\right)-u\left(\omega, \overline{\bar{x}}_{n, \delta}^{1}(w), \overline{\bar{x}}_{n, \delta}^{2}(w)\right)\right| d\left(\mu_{n}\right)_{\pi_{j}^{1}}(\omega)$

(by the definition of $\bar{x}_{n, \delta}^{1}$ and $\overline{\bar{x}}_{n, \delta}^{1}$ )

$=\sum_{k=t^{1}(j)}^{k=t^{1}(j)+K} \int_{\pi_{j}^{1} \cap \pi_{k}^{2}}\left|u\left(\omega, \bar{x}_{n, \delta}^{1}(w), \bar{x}_{n, \delta}^{2}(w)\right)-u\left(\omega, \bar{x}_{n, \delta}^{1}(w), \overline{\bar{x}}_{n, \delta}^{2}(w)\right)\right| d\left(\mu_{n}\right)_{\pi_{j}^{1}}(\omega)$ 
(by assumption (I) and the definition of $\bar{x}_{n, \delta}^{2}$ and $\overline{\bar{x}}_{n, \delta}^{2}$ )

$$
\begin{gathered}
\leq \sum_{k=t^{1}(j)}^{k=t^{1}(j)+K} \int_{\pi_{j}^{1} \cap \pi_{k}^{2}} C \delta\left(k-t^{1}(j)\right)\left(\left\|\widehat{x}_{0}^{2}(w)\right\|+\left\|\widehat{y}_{n}^{2}(w)\right\|\right) d\left(\mu_{n}\right)_{\pi_{j}^{1}}(\omega) \\
\leq 2 C \delta K^{2} \max _{s^{2} \in S^{2}}\left\|s^{2}\right\| .
\end{gathered}
$$

Similarly, it can be shown that

$$
\left|U_{\left(\mu_{n}\right)_{\pi_{j}^{1}}}\left(x^{1}, \bar{x}_{n, \delta}^{2}\right)-U_{\left(\mu_{n}\right)_{\pi_{j}^{1}}}\left(x^{1}, \overline{\bar{x}}_{n, \delta}^{2}\right)\right| \leq 2 C \delta K^{2} \max _{s^{2} \in S^{2}}\left\|s^{2}\right\| .
$$

Thus,

$$
\begin{aligned}
& U_{\left(\mu_{n}\right)_{\pi_{j}^{1}}}\left(x^{1}, \bar{x}_{n, \delta}^{2}\right)-U_{\left(\mu_{n}\right)_{\pi_{j}^{1}}}\left(\bar{x}_{n, \delta}^{1}, \bar{x}_{n, \delta}^{2}\right) \\
\leq & U_{\left(\mu_{n}\right)_{\pi_{j}^{1}}}\left(x^{1}, \overline{\bar{x}}_{n, \delta}^{2}\right)-U_{\left(\mu_{n}\right)_{\pi_{j}^{1}}}\left(\overline{\bar{x}}_{n, \delta}^{1}, \overline{\bar{x}}_{n, \delta}^{2}\right)+4 C \delta K^{2} \max _{s^{2} \in S^{2}}\left\|s^{2}\right\| .
\end{aligned}
$$

By the choice of $N(\delta), \overline{\bar{x}}_{n, \delta}^{i} \in \mathcal{O}_{\varepsilon^{\prime}}^{i}\left(\mu_{n}\right)$ for $i=1,2$ and every $n \geq N(\delta)$, being a convex combination of $\varepsilon^{\prime}$-optimal strategies. Therefore, by (8) in Remark 4 , for every $n \geq N(\delta)$

$$
U_{\left(\mu_{n}\right)_{\pi_{j}^{1}}}\left(x^{1}, \overline{\bar{x}}_{n, \delta}^{2}\right)-U_{\left(\mu_{n}\right)_{\pi_{j}^{1}}}\left(\overline{\bar{x}}_{n, \delta}^{1}, \overline{\bar{x}}_{n, \delta}^{2}\right) \leq \frac{\varepsilon^{\prime}}{\mu_{n}\left(\pi_{j}^{1}\right)} .
$$

From (23) and (24), for every $n \geq N(\delta)$

$$
U_{\left(\mu_{n}\right)_{\pi_{j}^{1}}}\left(x^{1}, \bar{x}_{n, \delta}^{2}\right)-U_{\left(\mu_{n}\right)_{\pi_{j}^{1}}}\left(\bar{x}_{n, \delta}^{1}, \bar{x}_{n, \delta}^{2}\right) \leq \frac{\varepsilon^{\prime}}{\mu_{n}\left(\pi_{j}^{1}\right)}+4 C \delta K^{2} \max _{s^{2} \in S^{2}}\left\|s^{2}\right\| \leq \varepsilon,
$$

by the definition of $\varepsilon^{\prime}$ and the choice of $\delta$ (see (16), (18)).

We conclude that (19) and (20) hold, and thus indeed $\left(\bar{x}_{n, \delta}^{1}, \bar{x}_{n, \delta}^{2}\right) \in$ $I B E_{\varepsilon}\left(\mu_{n}\right)$ for each $n \geq N(\delta)$.

It can be assumed w.l.o.g. that $\left\{N\left(\frac{\delta_{0}}{k}\right)\right\}_{k=1}^{\infty}$ is a strictly increasing sequence of positive integers (recall the definition of $\delta_{0}$ in (16)). Consider a sequence $\left\{\left(\hat{x}_{n}^{1}, \hat{x}_{n}^{2}\right)\right\}_{n=1}^{\infty} \subset X^{1} \times X^{2}$ defined by $\left(\hat{x}_{n}^{1}, \hat{x}_{n}^{2}\right) \equiv\left(\bar{x}_{n, \frac{\delta_{0}}{k}}^{1}, \bar{x}_{n, \frac{\delta_{0}}{k}}^{2}\right)$ if $N\left(\frac{\delta_{0}}{k}\right) \leq n<N\left(\frac{\delta_{0}}{k+1}\right)$ for $k=1,2, \ldots$, and $\left(\hat{x}_{n}^{1}, \hat{x}_{n}^{2}\right) \equiv\left(\widehat{y}_{n}^{1}, \widehat{y}_{n}^{2}\right)$ if $n<N\left(\delta_{0}\right)$. It follows from $(17)$ that $\lim _{n \rightarrow \infty}\left(\hat{x}_{n}^{1}, \hat{x}_{n}^{2}\right)=\left(\hat{x}_{0}^{1}, \hat{x}_{0}^{2}\right)$. Furthermore, since for 
every $0<\delta \leq \delta_{0}$ and every $n \geq N(\delta)$ it was shown that $\left(\bar{x}_{n, \delta}^{1}, \bar{x}_{n, \delta}^{2}\right) \in$ $I B E_{\varepsilon}\left(\mu_{n}\right)$, it follows that $\left(\hat{x}_{n}^{1}, \hat{x}_{n}^{2}\right) \in I B E_{\varepsilon}\left(\mu_{n}\right)$ for every $n \geq 1$.

When at least one player has a finite number of types, ALSC obtains without assuming either (I) or (II) of Proposition 3. Convergence of common priors implies, in this case, almost uniform convergence of conditional beliefs, and thus the IBE expected payoff correspondence is ALSC (for general, not just the zero-sum, games), according to the main result of Kajii and Morris (1994, 1998). The ALSC of the IBE strategy correspondence transpires from the proof of that result. For the sake of completeness, however, we state and prove the following proposition, for zero-sum Bayesian games with a finite number of types for at least one player.

Proposition 4. Assume $\min \left(T^{1}, T^{2}\right)<\infty$. Let $\left\{\mu_{n}\right\}_{n=0}^{\infty} \subset \Delta(\Omega, \mathcal{F})$ be such that $\lim _{n \rightarrow \infty} \mu_{n}=\mu_{0}$, and $\left(\mu_{0}\right)\left(\pi_{j}^{i}\right)>0$ for every $i=1,2$ and every 1 $\leq j<\min \left(T^{i}+1, \infty\right)$. Then the IBE correspondence is ALSC along $\left\{\mu_{n}\right\}_{n=1}^{\infty}$.

Proof. Assume w.l.o.g. that $T^{1}<\infty$. Let $\left\{\mu_{n}\right\}_{n=0}^{\infty} \subset \Delta(\Omega, \mathcal{F})$ be a sequence satisfying all the assumptions, and fix $\left(\hat{x}_{0}^{1}, \hat{x}_{0}^{2}\right) \in I B E\left(\mu_{0}\right), \varepsilon>0$. Also consider a sequence $\left\{y_{n}^{2}\right\}_{n=1}^{\infty} \subset X^{2}$ such that $y_{n}^{2}$ is an (ex-ante, or, equivalently, interim) best response of player 2 to the strategy $\hat{x}_{0}^{1}$ of player 1 in the game $G\left(\mu_{n}\right)$, for each $n \geq 1$.

Let

$$
0<\delta \leq \delta_{0} \equiv \frac{\varepsilon}{16 M} \min _{1 \leq j \leq T^{1}} \mu_{0}\left(\pi_{j}^{1}\right),
$$

and let an integer $0<J(\delta)<\min \left(T^{2}+1, \infty\right)$ be such that $\mu\left(\bigcup_{j=1}^{J(\delta)} \pi_{j}^{2}\right)>1-\delta$. Define a sequence $\left\{\left(\bar{x}_{n, \delta}^{2}\right)\right\}_{n=1}^{\infty} \subset X^{2}$ as follows: for every $n \geq 1$,

$$
\bar{x}_{n, \delta}^{2}(\omega) \equiv\left\{\begin{array}{lc}
\hat{x}_{0}^{2}(\omega), & \text { if } \omega \in \bigcup_{j=1}^{J(\delta)} \pi_{j}^{2} \\
y_{n}^{2}(\omega), & \text { otherwise. }
\end{array}\right.
$$


The strategy $\hat{x}_{0}^{1}$, being in $\mathcal{O}^{1}\left(\mu_{0}\right)$ by Remarks 2 and 3 , is also in $\mathcal{O}_{4 M d\left(\mu_{n}, \mu_{0}\right)}^{1}\left(\mu_{n}\right)$ by Proposition 2 , and thus, for every $1 \leq j \leq T^{1}$ with $\mu_{n}\left(\pi_{j}^{1}\right)>0$ and every $x^{1} \in X^{1}$,

$$
U_{\left(\mu_{n}\right)_{\pi_{j}^{1}}}\left(\hat{x}_{0}^{1}, \hat{x}_{0}^{2}\right) \geq U_{\left(\mu_{n}\right)_{\pi_{j}^{1}}}\left(x^{1}, \hat{x}_{0}^{2}\right)-\frac{4 M d\left(\mu_{n}, \mu_{0}\right)}{\mu_{n}\left(\pi_{j}^{1}\right)}
$$

by (8) in Remark 4. Since clearly, for every such $j$ and $x^{1}$

$$
\begin{gathered}
\left|U_{\left(\mu_{n}\right)_{\pi_{j}^{1}}}\left(x^{1}, \hat{x}_{0}^{2}\right)-U_{\left(\mu_{n}\right)_{\pi_{j}^{1}}}\left(x^{1}, \bar{x}_{n, \delta}^{2}\right)\right| \\
\leq 2 M\left(\mu_{n}\right)_{\pi_{j}^{1}}\left(\Omega \backslash \bigcup_{j=1}^{J(\delta)} \pi_{j}^{2}\right) \leq \frac{2 M \delta}{\mu_{n}\left(\pi_{j}^{1}\right)},
\end{gathered}
$$

(28) implies that

$$
U_{\left(\mu_{n}\right)_{\pi_{j}^{1}}}\left(\hat{x}_{0}^{1}, \bar{x}_{n, \delta}^{2}\right) \geq U_{\left(\mu_{n}\right)_{\pi_{j}^{1}}}\left(x^{1}, \bar{x}_{n, \delta}^{2}\right)-\frac{4 M\left(d\left(\mu_{n}, \mu_{0}\right)+\delta\right)}{\mu_{n}\left(\pi_{j}^{1}\right)} .
$$

By our assumptions on $\left\{\mu_{n}\right\}_{n=0}^{\infty}$, there exists $N_{1}(\delta)>0$ such that $d\left(\mu_{n}, \mu_{0}\right)$ $\leq \delta_{0}$ and $\min _{1 \leq j \leq T^{1}} \mu_{n}\left(\pi_{j}^{1}\right) \geq \frac{1}{2} \min _{1 \leq j \leq T^{1}} \mu_{0}\left(\pi_{j}^{1}\right)$ for every $n \geq N_{1}(\delta)$. The choice of $\delta$ in (26) and the inequality (29) guarantee that

$$
U_{\left(\mu_{n}\right)_{\pi_{j}^{1}}}\left(\hat{x}_{0}^{1}, \bar{x}_{n, \delta}^{2}\right) \geq U_{\left(\mu_{n}\right)_{\pi_{j}^{1}}}\left(x^{1}, \bar{x}_{n, \delta}^{2}\right)-\varepsilon
$$

for every $1 \leq j \leq T^{1}$, every $x^{1} \in X^{1}$, and every $n \geq N_{1}(\delta)$.

Just as in (28), for every $1 \leq j \leq J(\delta)$ with $\mu_{n}\left(\pi_{j}^{2}\right)>0$ and $x^{2} \in X^{2}$

$$
U_{\left(\mu_{n}\right)_{\pi_{j}^{2}}}\left(\hat{x}_{0}^{1}, \hat{x}_{0}^{2}\right) \leq U_{\left(\mu_{n}\right)_{\pi_{j}^{2}}}\left(\hat{x}_{0}^{1}, x^{2}\right)+\frac{4 M d\left(\mu_{n}, \mu_{0}\right)}{\mu_{n}\left(\pi_{j}^{2}\right)} .
$$

By our assumptions on $\left\{\mu_{n}\right\}_{n=1}^{\infty}$, there exists $N_{2}(\delta)>0$ such that $\min _{1 \leq j \leq J(\delta)} \mu_{n}\left(\pi_{j}^{2}\right)$ $>0$ and $\max _{1 \leq j \leq J(\delta)} \frac{4 M d\left(\mu_{n}, \mu_{0}\right)}{\mu_{n}\left(\pi_{j}^{2}\right)} \leq \varepsilon$ for every $n \geq N_{2}(\delta)$, and therefore for every $1 \leq j \leq J(\delta)$, every $x^{2} \in X^{2}$, and every $n \geq N_{2}(\delta)$

$$
U_{\left(\mu_{n}\right)_{\pi_{j}^{2}}}\left(\hat{x}_{0}^{1}, \hat{x}_{0}^{2}\right) \leq U_{\left(\mu_{n}\right)_{\pi_{j}^{2}}}\left(\hat{x}_{0}^{1}, x^{2}\right)+\varepsilon .
$$

But by $(27), \hat{x}_{0}^{1}(\omega)=\bar{x}_{n, \delta}^{2}(\omega)$ for all $\omega \in \pi_{j}^{2}$, and thus in fact the following inequality holds:

$$
U_{\left(\mu_{n}\right)_{\pi_{j}^{2}}}\left(\hat{x}_{0}^{1}, \bar{x}_{n, \delta}^{2}\right) \leq U_{\left(\mu_{n}\right)_{\pi_{j}^{2}}}\left(\hat{x}_{0}^{1}, x^{2}\right)+\varepsilon .
$$


When $j>J(\delta), \bar{x}_{n, \delta}^{2}(\omega)=y_{n}^{2}(\omega)$ for all $\omega \in \pi_{j}^{2}$, and by the definition of $\left\{y_{n}^{2}\right\}_{n=1}^{\infty}$ as the sequence of best responses to $\hat{x}_{0}^{1}$ in the games $\left\{G\left(\mu_{n}\right)\right\}_{n=1}^{\infty}$,

$$
U_{\left(\mu_{n}\right)_{\pi_{j}^{2}}}\left(\hat{x}_{0}^{1}, \bar{x}_{n, \delta}^{2}\right) \leq U_{\left(\mu_{n}\right)_{\pi_{j}^{2}}}\left(\hat{x}_{0}^{1}, x^{2}\right)
$$

for every $J(\delta) \leq j<\min \left(T^{2}+1, \infty\right)$, every $x^{2} \in X^{2}$ and every $n \geq N_{2}(\delta)$. Thus, (32) in fact holds for every $1 \leq j<\min \left(T^{2}+1, \infty\right)$, every $x^{2} \in X^{2}$, and every $n \geq N_{2}(\delta)$. This fact, coupled with (30), shows that $\left(\hat{x}_{0}^{1}, \bar{x}_{n, \delta}^{2}\right) \in$ $I B E_{\varepsilon}\left(\mu_{n}\right)$ for each $n \geq N(\delta) \equiv \max \left(N_{1}(\delta), N_{2}(\delta)\right)$.

Since $\lim _{\delta \rightarrow 0} \bar{x}_{n, \delta}^{2}=\hat{x}_{0}^{2}$, the construction in the last paragraph of the proof of proposition 3 can be repeated to create out of $\left\{\bar{x}_{n, \delta}^{2}\right\}_{n=1}^{\infty}$ a sequence $\left\{\hat{x}_{n}^{2}\right\}_{n=1}^{\infty} \subset X^{2}$ such that $\lim _{n \rightarrow \infty}\left(\hat{x}_{0}^{1}, \hat{x}_{n}^{2}\right)=\left(\hat{x}_{0}^{1}, \hat{x}_{0}^{2}\right)$, and $\left(\hat{x}_{0}^{1}, \hat{x}_{n}^{2}\right) \in$ $I B E_{\varepsilon}\left(\mu_{n}\right)$ for every $n \geq 1$. 


\section{References}

1. Einy E., O. Haimanko, D. Moreno, and B. Shitovitz. (2008). "Uniform Continuity of the Value of Zero-Sum Games with Differential Information," Mathematics of Operations Research 33, 552-560.

2. Engl, G. (1995). "Lower Hemicontinuity of the Nash Equilibrium Correspondence", Games and Economic Behavior 9, 151-160.

3. Gossner, O. and J.-F. Mertens (2001). "The Value of Information in Zero-Sum Games," mimeo.

4. Kajii, A., and S. Morris. (1994) "Payoff Continuity in Incomplete Information Games and Almost Uniform Convergence of Beliefs," CARESS Working paper \# 94-17.

5. Kajii, A., and S. Morris (1998). "Payoff Continuity in Incomplete Information Games," Journal of Economic Theory 82, 267-276.

6. Lehrer, E. and D. Rosenberg (2006). " "What restrictions do Bayesian games impose on the value of information?" Journal of Mathematical Economics 42, pp. 343-357.

7. Milgrom, P. and R.J. Weber (1985). "Distributional Strategies for Games with Incomplete Information," Mathematics of Operations Research 10, pp. 619-632.

8. Monderer, D. and D. Samet (1996). "Proximity of Information in Games with Common Beliefs," Mathematics of Operations Research 21, pp. 707-725.

9. Rubinstein, A., (1989). "The Electronic Mail Game: Strategic Behavior Under "Almost Common Knowledge", American Economic Review 79, pp. 385-391.

10. Shiryaev, A. N. (1996). Probability, Second Edition. Graduate Texts in Mathematics, Springer-Verlag.

11. Sion, M. (1958). "On General Minimax Theorem," Pacific Journal of Mathematics 8, 171-176. 
12. Sorin, S. (2002). A First Course on Zero-Sum Repeated Games. Mathematiques and Applications, Vol. 37. Springer-Verlag. 